(2) Open Access Full Text Article

ORIGINALRESEARCH

\title{
Association of Urinary Polycyclic Aromatic Hydrocarbons and Diabetes in Korean Adults: Data from the Korean National Environmental Health Survey Cycle 2 (20I2-2014)
}

This article was published in the following Dove Press journal: Diabetes, Metabolic Syndrome and Obesity: Targets and Therapy

\author{
Yon Ju Nam (D) \\ Shin-Hye Kim (iD) ${ }^{2}$ \\ 'College of Health Science, Korea \\ University, Seoul 0284I, Korea; \\ ${ }^{2}$ Department of Pediatrics, Sanggye Paik \\ Hospital, Inje University College of \\ Medicine, Seoul 01757, Korea
}

Purpose: To investigate the associations between the urinary levels of polycyclic aromatic hydrocarbons (PAHs) and diabetes mellitus in Korean adults.

Materials and Methods: We examined the data of 6478 participants aged $\geq 19$ years from the Korean National Environmental Health Survey (KoNEHS) cycle 2 (2012-2014). The urinary levels of 1-hydroxypyrene (1-OHP), 2-naphthol (2-NAP), 1-hydroxyphenathrene (1-OHPhe), and 2-hydroxyfluorene (2-OHFlu) were measured by gas chromatographymass spectrometry. Diabetes mellitus was defined as a self-report of physician-diagnosed diabetes mellitus or the use of oral hypoglycemics or insulin. Analyses were adjusted for sex, age, body mass index, household income, alcohol consumption, physical activity, urinary creatinine and cotinine, menopausal status, and quartiles of all other PAHs.

Results: The prevalence of diabetes was $6.5 \%$ in the study population. In men, the geometric means of the 2-NAP and 2-OHFlu levels were higher in participants with diabetes mellitus than in those without diabetes mellitus [4.11 vs $3.26 \mu \mathrm{g} / \mathrm{L}(P<0.05)$ and $0.45 \mathrm{vs} 0.40$ $\mu \mathrm{g} / \mathrm{L}(P<0.05)$, respectively]. In women, the geometric mean of 2-NAP levels was also higher in participants with diabetes mellitus than in those without diabetes mellitus (1.81 vs $0.56 \mu \mathrm{g} / \mathrm{L}, P<0.05)$, but there were no significant differences in geometric means for other PAHs. A higher odds ratio (OR) of diabetes was found in participants with the highest quartiles of urinary 2-NAP [OR 1.83, 95\% confidence interval (CI) 1.29-2.60] and 2-OHFlu (OR $1.81,95 \%$ CI $1.10-2.98$ ) than in those with the lowest quartiles.

Conclusion: The urinary 2-NAP and 2-OHFlu levels were associated with diabetes mellitus in Korean adults. Further studies are needed to determine a potential causal relationship between PAH exposure and diabetes mellitus and its underlying mechanism.

Keywords: environmental pollutant, 2-naphthol, 2-hydroxyfluorene, environmental exposure, health survey, endocrine disruptors

\section{Introduction}

Over the last few decades, the prevalence of type 2 diabetes has significantly increased worldwide. ${ }^{1}$ The prevalence of diabetes is predicted to reach 693 million individuals by $2045,{ }^{2}$ and especially in Korea, it has increased astonishingly among adults over the past 15 years. ${ }^{3}$ In addition to the well-known risk factors of diabetes, including genetic predisposition and obesity, ${ }^{4}$ recent reports have indicated that endocrine-disrupting chemicals (EDCs) may play a significant
Correspondence: Shin-Hye Kim Department of Pediatrics, Inje University Sanggye Paik Hospital, Inje University

College of Medicine, I342 Dongil-Ro,

Nowon-Gu, Seoul 01757, Korea

Tel +82-2-950-4812

Fax+82-2-950-1246

Email S2635@paik.ac.kr
Diabetes, Metabolic Syndrome and Obesity: Targets and Therapy 2020:1 3 3993-4003

3993 
role in the development of diabetes. ${ }^{1}$ Among EDCs, polycyclic aromatic hydrocarbons (PAHs) have been extensively studied recently.

PAHs are a group of environmental pollutants whose structures contain aromatic rings. They are generated by incomplete combustion of organic materials, such as coal, gasoline, and wood. The major sources of PAH exposure include inhalation of cigarette smoke, vehicle exhaust, and processed fossil fuels. It could also be derived from the ingestion of grilled and charred meats, contaminated food products, and the cooking process. ${ }^{5}$

In experimental studies, the possible mechanisms linking PAH exposure to diabetes include oxidative stress ${ }^{6}$ and systemic inflammation, ${ }^{7,8}$ which induce $\beta$-cell dysfunction ${ }^{9}$ and disruption of the insulin signaling pathway. ${ }^{10}$

Previous epidemiologic studies from the USA ${ }^{11-14}$ and China ${ }^{15-18}$ have shown a positive association between PAH exposure determined by the urinary PAH levels and diabetes. However, no studies on this issue have been conducted using nationwide data except for the US National Health and Nutrition Examination Survey (NHANES). Severe air pollution in Korea has also become an increasing threat. ${ }^{19}$ Given the rapidly increasing prevalence of diabetes in the Korean population, ${ }^{3}$ epidemiologic studies are needed to elucidate the link between PAH exposure and diabetes in Korean adults.

In this study, we aimed to evaluate the associations between the urinary PAH levels and diabetes using nationally representative data from the Korean National Environmental Health Survey (KoNEHS).

\section{Materials and Methods Study Population}

The KoNEHS is a cross-sectional nationwide biomonitoring survey, which aims to monitor the exposure of people to environmental chemicals. It involves a stratified multistage probability sample representative of the entire Korean population. The survey collects data on socioeconomic status and health conditions and conducts questionnaire-based personal interviews on environmental chemical exposure, physical examinations, and laboratory tests. A detailed description of the KoNEHS study design was provided in a previous study. ${ }^{20}$

We specifically used data from the second cycle of the KoNEHS, which was conducted between 2012 and 2014. A total of 6478 participants aged $\geq 19$ years with data on urinary levels of 1-hydroxypyrene (1-OHP), 2-naphthol
(2-NAP), 1-hydroxyphenathrene (1-OHPhe), and 2-hydroxyfluorene (2-OHFlu) were included for analysis. Diabetes was defined as a self-report of physician-diagnosed diabetes or the current oral hypoglycemic medication or insulin use at the time of the interview.

The KoNEHS was conducted in accordance with the Declaration of Helsinki. All participants of the KoNEHS agreed to participate the survey and signed a written informed consent. The study protocol was approved by the Institutional Review Board of Inje University Sanggye Paik Hospital (approval number: SGPAIK-202008-001).

\section{Measurement of Urinary PAHs}

Random spot urine samples were collected and stored within $24 \mathrm{~h}$ following the standard procedures established by the National Institute of Environmental Research. The urinary levels of PAHs were analyzed by gas chromatography-mass spectrometry (Clarus 680T, PerkinElmer, Waltham, MA, USA).

For external quality control, all institutions involved in the analysis participated in the German G-EQUAS and the Special Health Quality Control program by the Occupational Safety and Health Research Institute. All institutions passed both programs. Internal quality control was conducted following the guidelines of the National Institute of Environmental Research using G-EQUAS, Bio-Rad, ClinCheck I and II, and NIST standards. The limit of detection (LOD) values for 1-OHP, 2-NAP, 1-OHPhe, and 2-OHFlu were $0.015,0.050,0.047$, and $0.040 \mu \mathrm{g} / \mathrm{L}$, respectively. Values below the LOD were calculated as the method detection limit divided by $\sqrt{2}$. All urinary PAH levels were adjusted for the urinary creatinine levels.

\section{Study Covariates}

Information on age, sex, household income, smoking, alcohol consumption, physical activity, and menopausal status (in women) was obtained using standardized questionnaires during the survey interview. Monthly household income of the participants [in US dollars (USD)] was grouped into four categories: <920, 920-2090, 2090-3400, and $\geq 3400$ USD. Former or current smokers were active smokers who had smoked at least 100 cigarettes in their lifetime. Former smokers were defined as those who had quit smoking at the time of the interview. The participants who had consumed at least one glass of alcohol every month over the past year were defined as 
former or current drinkers. Former drinkers were defined as those who had quit drinking at the time of the interview. Regular physical activity was indicated as "yes" when the participant engaged in moderate and severe exercise on a regular basis for $>30 \mathrm{~min}$ at a time and more than three times/week.

Body mass index (BMI) was calculated as weight $(\mathrm{kg})$ divided by the square of height $\left(\mathrm{m}^{2}\right)$. The participants were classified as underweight $(<18.5)$, normal weight $(18.5-$ $22.9)$, overweight (23-24.9), and obese ( $\geq 25)$ according to the Asian BMI classifications. ${ }^{21}$

\section{Statistical Analysis}

Statistical analyses were performed using SPSS version 25.0 (IBM Corp., Armonk, NY, USA). Sampling weights were used for the complex sampling model for all analyses. The demographic characteristics and health behaviors of participants were analyzed using the complex samples general linear model and cross-tabulation procedures. The complex samples descriptive procedure was used to calculate the geometric means (GMs) and 95\% confidence intervals (95\% CIs) of the PAH levels. The GMs of the urinary PAH levels were compared in terms of the demographic and behavioral factors of diabetes using the complex samples general linear model after adjusting for the log-transformed urinary creatinine and cotinine levels. The urinary PAH levels were logtransformed due to their skewed distribution. Complex samples logistic regression analyses were used to calculate the odds ratios (ORs) and 95\% CIs for diabetes, according to the PAH quartiles, after adjusting the multiple covariates for sex, age, BMI, household income, alcohol consumption, physical activity, log-transformed urinary creatinine and cotinine, and menopausal status (in women). For all analyses, $P$ values were two-tailed, and a $P$ value $<0.05$ was considered statistically significant.

\section{Results}

\section{General Characteristics of Participants}

Table 1 presents the general characteristics of the study participants. The mean age was $46.3 \pm 0.4$ years, and $42.8 \%$ of participants were men. The proportions of overweight and obese participants were significantly higher in men $(25.1 \%$ and $43.3 \%)$ than in women $(20.9 \%$ and $32.0 \%$ ). Smoking prevalence was significantly higher in men than in women $(39.8 \%$ vs $3.8 \%, P<0.001)$. The overall prevalence of diabetes was $6.5 \%$, which was not significantly different by sex.

\section{Distributions of PAHs According to the Participant Characteristics}

Table 2 summarizes the urinary PAH levels according to the participant characteristics. The proportion of participants who had a detectable level (above the LOD) of each urinary $\mathrm{PAH}$ was $97.1 \%, 98.8 \%, 79.0 \%$, and $92.9 \%$ for 1-OHP, 2-NAP, 1-OHPhe, and 2-OHFlu, respectively. The GMs of the urinary levels of 1-OHP, 2-NAP, 1-OHPhe, and $2-\mathrm{OHFlu}$ were $0.15,2.23,0.10$, and $0.27 \mu \mathrm{g} / \mathrm{L}$, respectively, and were significantly higher in men than in women. Moreover, the GMs of all urinary PAH levels were significantly higher in former/current smokers than in never smokers.

Table 3 shows the adjusted GMs of the urinary PAH levels in men. The GM values were significantly higher in those aged $\geq 40$ years than in those aged $<40$ years $(P<$ $0.05)$. In men, the GM of 2-OHFlu levels was significantly higher in the highest-BMI than in the normal-BMI group. There were no significant differences in other PAH levels according to the BMI status. There were also no significant differences in all PAH levels according to the household income and the alcohol consumption status. Meanwhile, the GM of the 1-OHPhe levels was significantly higher in participants who performed regular physical activity than in those who did not $(P<0.05)$, but there were no significant differences with other PAHs. The GMs of the urinary 2-NAP and 2-OHFlu levels were significantly higher in participants with diabetes mellitus than in those without (4.11 vs $3.26 \mu \mathrm{g} / \mathrm{L}$ and 0.45 vs $0.40 \mu \mathrm{g} / \mathrm{L}$, respectively; all $P<0.05$ ).

Table 4 presents the adjusted GMs of the urinary PAH levels in women. The GMs of the 1-OHP, 1-OHPhe, and 1-OHFlu levels were significantly higher in the lowestBMI than in the normal-BMI group $(P<0.05)$, whereas the GMs of 1-OHP and 1-OHPhe levels were significantly higher in the highest-BMI than in the normal-BMI group $(P<0.05)$. No significant differences in the PAH levels were observed in women based on the household income, alcohol consumption, physical activity, and menopausal status. Women with diabetes mellitus had a higher GM of the urinary 2-NAP levels than women without (1.81 vs $0.56 \mu \mathrm{g} / \mathrm{L}, P<0.05)$. Regarding other urinary PAHs in women, no significant differences in GM values were found between those with and without diabetes mellitus. 
Table I General Characteristics of the Study Subjects

\begin{tabular}{|c|c|c|c|c|c|c|c|c|c|c|}
\hline \multirow[b]{2}{*}{ Age (years) } & \multicolumn{3}{|c|}{ Total $(n=6478)$} & \multicolumn{3}{|c|}{ Men $(n=2774)$} & \multicolumn{3}{|c|}{ Women $(n=3704)$} & \multirow{2}{*}{$\begin{array}{l}\boldsymbol{P} \text {-value } \\
<0.001\end{array}$} \\
\hline & 46.3 & \pm & 0.4 & 45.4 & \pm & 0.4 & 47.1 & \pm & 0.5 & \\
\hline Age group (n,\%) & & & & & & & & & & $<0.001$ \\
\hline $19-29 \mathrm{yr}$ & 537 & & $17.7 \%$ & 263 & & $18.5 \%$ & 274 & & $16.9 \%$ & \\
\hline $30-39 \mathrm{yr}$ & 1056 & & $19.9 \%$ & 435 & & $20.7 \%$ & 621 & & $19.1 \%$ & \\
\hline $40-49 \mathrm{yr}$ & 1226 & & $21.4 \%$ & 504 & & $22.0 \%$ & 722 & & $20.8 \%$ & \\
\hline $50-59 \mathrm{yr}$ & 1439 & & $19.4 \%$ & 578 & & $19.7 \%$ & 861 & & $19.1 \%$ & \\
\hline $60-69 \mathrm{yr}$ & 1335 & & $11.1 \%$ & 597 & & $10.8 \%$ & 738 & & $11.3 \%$ & \\
\hline$\geq 70 \mathrm{yr}$ & 885 & & $10.6 \%$ & 397 & & $8.3 \%$ & 488 & & $12.8 \%$ & \\
\hline BMI group $(n, \%)$ & & & & & & & & & & $<0.001$ \\
\hline Underweight & 154 & & $3.0 \%$ & 48 & & $1.6 \%$ & 106 & & $4.40 \%$ & \\
\hline Normal & 2183 & & $36.4 \%$ & 809 & & $29.9 \%$ & 1374 & & $42.80 \%$ & \\
\hline Overweight & 1600 & & $23.0 \%$ & 738 & & $25.1 \%$ & 862 & & $20.90 \%$ & \\
\hline Obese & 2541 & & $37.6 \%$ & 1179 & & $43.4 \%$ & 1362 & & $32.00 \%$ & \\
\hline Household income (USD/months) & & & & & & & & & & $<0.001$ \\
\hline$<920$ USD & 1629 & & $17.3 \%$ & 639 & & $14.6 \%$ & 990 & & $19.9 \%$ & \\
\hline 920-2090 USD & 1396 & & $19.1 \%$ & 615 & & $19.0 \%$ & 781 & & $19.2 \%$ & \\
\hline $2090-3400$ USD & 1488 & & $26.4 \%$ & 660 & & $27.5 \%$ & 828 & & $25.4 \%$ & \\
\hline$\geq 3400$ USD & 1965 & & $37.2 \%$ & 860 & & $38.9 \%$ & 1105 & & $35.5 \%$ & \\
\hline Smoking $(n, \%)$ & & & & & & & & & & $<0.001$ \\
\hline Never & 4259 & & $62.7 \%$ & 762 & & $30.6 \%$ & 3497 & & $93.9 \%$ & \\
\hline Former & 1058 & & $15.8 \%$ & 990 & & $29.6 \%$ & 68 & & $2.3 \%$ & \\
\hline Current & 1161 & & $21.5 \%$ & 1022 & & $39.8 \%$ & 139 & & $3.8 \%$ & \\
\hline Drinking $(n, \%)$ & & & & & & & & & & $<0.001$ \\
\hline Never & 2230 & & $29.7 \%$ & 447 & & $15.30 \%$ & 1783 & & $43.6 \%$ & \\
\hline Former & 424 & & $5.6 \%$ & 282 & & $7.00 \%$ & 142 & & $4.2 \%$ & \\
\hline Current & 3824 & & $64.7 \%$ & 2045 & & $77.60 \%$ & 1779 & & $52.2 \%$ & \\
\hline Regular physical activity (n,\%) & & & & & & & & & & $<0.001$ \\
\hline No & 4658 & & $72.3 \%$ & 1931 & & $69.3 \%$ & 2727 & & $75.1 \%$ & \\
\hline Yes & 1820 & & $27.7 \%$ & 843 & & $30.7 \%$ & 977 & & $24.9 \%$ & \\
\hline Diabetes mellitus ( $\mathrm{n}, \%)$ & & & & & & & & & & 0.482 \\
\hline No & 5928 & & $93.5 \%$ & 2504 & & $93.3 \%$ & 3424 & & $93.8 \%$ & \\
\hline Yes & 550 & & $6.5 \%$ & 270 & & $6.7 \%$ & 280 & & $6.2 \%$ & \\
\hline
\end{tabular}

Notes: Mean \pm SE or number (\%), BMI $<18.5$ for underweight, 18.5-22.9 for normal weight, 23-24.9 for overweight, $\geq 25$ for obese.

Abbreviations: BMI,body mass index; USD, US dollars.

\section{Association Between the Urinary PAH Levels and Diabetes}

Adjusted ORs and 95\% CIs for diabetes mellitus according to the quartiles of urinary PAHs are presented in Table 5. The participants in the highest 2-NAP quartile had a higher OR for diabetes than those in the lowest quartile (OR 1.83, 95\% CI 1.29-2.60). The adjusted ORs for diabetes across quartiles of 2-NAP were 1, 1.08, 1.57, and $1.83(P<0.001)$. Conversely, the OR for diabetes was higher in participants in the highest than in the lowest 2-OHFlu quartile (OR 1.81, 95\% CI 1.10-2.98). However, this association was not statistically significant in the subgroup analysis by sex.

\section{Discussion}

In this study, using national representative data, we demonstrated that Korean adults with diabetes mellitus had higher urinary levels of 2-NAP in both sexes than those without. We also presented that men had higher urinary 2-OHFlu levels than women. Moreover, quartiles of urinary 2-NAP levels were positively associated with OR for diabetes in a dose-dependent manner in both sexes 
Table 2 Distributions of Urinary PAHs Metabolites Concentrations

\begin{tabular}{|c|c|c|c|c|c|c|c|}
\hline & \multirow[b]{2}{*}{$\mathbf{N}$} & \multirow{2}{*}{$\begin{array}{l}\text { GM }(95 \% \mathrm{Cl}) \\
\text { Crude }\end{array}$} & \multirow[b]{2}{*}{$\% \geq$ LOD } & \multicolumn{4}{|c|}{ Selected Percentiles } \\
\hline & & & & 25th & 50th & 75th & 95th \\
\hline \multicolumn{8}{|c|}{ I-hydroxypyrene ( $\mu \mathrm{g} / \mathrm{L})$} \\
\hline Total & 6418 & $0.15(0.14-0.16)$ & 97.1 & 0.09 & 0.14 & 0.25 & 0.60 \\
\hline $\operatorname{Men}^{\mathrm{a}}$ & 2745 & $0.18(0.17-0.19)$ & 98.1 & 0.1 & 0.18 & 0.32 & 0.76 \\
\hline Women & 3673 & $0.13(0.12-0.14)^{* * *}$ & 96.4 & 0.08 & 0.13 & 0.21 & 0.56 \\
\hline \multicolumn{8}{|c|}{ Smoking status } \\
\hline Never $^{\mathrm{a}}$ & 4226 & $0.12(0.12-0.13)$ & 96.4 & 0.08 & 0.13 & 0.21 & 0.49 \\
\hline Former & 1046 & $0.15(0.14-0.16)^{* * *}$ & 97.7 & 0.09 & 0.14 & 0.23 & 0.57 \\
\hline Current & 1146 & $0.27(0.25-0.28)^{* * * *}$ & 99.2 & 0.16 & 0.28 & 0.44 & 0.9 \\
\hline \multicolumn{8}{|c|}{ 2-naphthol ( $\mu g / L)$} \\
\hline Total & 6410 & $2.23(2.11-2.35)$ & 98.8 & 0.83 & 1.91 & 5.32 & 19.9 \\
\hline $\operatorname{Men}^{\mathrm{a}}$ & 2750 & $3.02(2.82-3.25)$ & 99.3 & 1.09 & 2.93 & 8.5 & 23.1 \\
\hline Women & 3660 & $1.64(1.54-1.76)^{* * * *}$ & 98.4 & 0.71 & 1.46 & 3.53 & 14.3 \\
\hline \multicolumn{8}{|c|}{ Smoking status } \\
\hline Never $^{\mathrm{a}}$ & 4210 & $1.52(\mid .43-1.61)$ & 98.5 & 0.68 & 1.39 & 3.23 & 12.6 \\
\hline Former & 1049 & $1.76(1.60-1.92)^{* *}$ & 98.7 & 0.8 & 1.66 & 3.85 & 17.1 \\
\hline Current & $|15|$ & $7.93(7.39-8.52)^{* * * *}$ & 100.0 & 4.51 & 8.8 & 14.7 & 30.7 \\
\hline \multicolumn{8}{|c|}{ I-hydroxyphenanthrene ( $\mu g / L)$} \\
\hline Total & 6413 & $0.10(0.09-0.10)$ & 79.0 & 0.05 & 0.09 & 0.16 & 0.38 \\
\hline $\operatorname{Men}^{\mathrm{a}}$ & 2746 & $0.11(0.10-0.11)$ & 82.9 & 0.06 & 0.11 & 0.19 & 0.45 \\
\hline Women & 3667 & $0.09(0.08-0.09)^{* * *}$ & 76.1 & 0.05 & 0.08 & 0.14 & 0.39 \\
\hline \multicolumn{8}{|c|}{ Smoking status } \\
\hline Never $^{\mathrm{a}}$ & 4220 & $0.08(10.08-0.09)$ & 75.8 & 0.05 & 0.08 & 0.14 & 0.33 \\
\hline Former & 1047 & $0.09(0.09-0.10)^{*}$ & 78.4 & 0.05 & 0.09 & 0.16 & 0.40 \\
\hline Current & 1146 & $0.14(0.13-0.15)^{* * *}$ & 91.5 & 0.09 & 0.14 & 0.24 & 0.48 \\
\hline \multicolumn{8}{|c|}{ 2-hydroxyfluorene $(\mu g / L)$} \\
\hline Total & 6397 & $0.27(0.25-0.28)$ & 92.9 & 0.12 & 0.24 & 0.51 & 1.86 \\
\hline $\operatorname{Men}^{\mathrm{a}}$ & 2735 & $0.40(0.37-0.43)$ & 95.7 & 0.17 & 0.38 & 1.00 & 2.38 \\
\hline Women & 3662 & $0.18(0.17-0.19)^{* * * *}$ & 90.8 & 0.09 & 0.19 & 0.35 & 1.00 \\
\hline \multicolumn{8}{|c|}{ Smoking status } \\
\hline Never $^{\mathrm{a}}$ & 4216 & $0.18(0.17-0.19)$ & 91.1 & 0.10 & 0.19 & 0.33 & 0.80 \\
\hline Former & 1045 & $0.22(0.20-0.24)^{* * *}$ & 93.6 & 0.12 & 0.23 & 0.42 & 1.11 \\
\hline Current & 1136 & $1.01(0.93-1.09)^{* * * *}$ & 99.1 & 0.62 & 1.13 & 1.82 & 3.32 \\
\hline
\end{tabular}

Notes: ${ }^{a}$ Reference for comparison of geometric mean in each variable. $* * * P<0.001, * * P<0.01, * P<0.05$.

Abbreviations: BMI, body mass index; $\mathrm{Cl}$, confidence interval; $\mathrm{GM}$, geometric mean; LOD, limit of detection.

after considering all covariates, including other PAH levels. The positive association between the urinary 2-OHFlu levels and diabetes was weak and lost its statistical significance in the sex-specific analysis. To our knowledge, this is the first large-scale nationwide epidemiologic study showing the relationship between urinary PAHs and diabetes in the non-Western population.
We found that the urinary levels of all four PAHs in this study were significantly higher in men than in women and higher in former/current smokers than in never smokers. These findings were consistent with those of previous studies based on the US NHANES ${ }^{22}$ and the Canadian Health Measure Survey. ${ }^{23}$ Since PAHs are produced during the incomplete combustion of organic material, the 
Table 3 Adjusted Geometric Mean of Polycyclic Aromatic Hydrocarbons ( $\mu \mathrm{g} / \mathrm{L})$ Stratified by Participants Characteristics in Men

\begin{tabular}{|c|c|c|c|c|}
\hline \multirow[t]{2}{*}{ Factors } & \multicolumn{4}{|c|}{ Adjusted Geometric Mean $(95 \% \mathrm{Cl})$} \\
\hline & $\begin{array}{l}\text { I-hydroxypyrene } \\
(\mu \mathrm{g} / \mathrm{L})\end{array}$ & 2-naphthol $(\mu \mathrm{g} / \mathrm{L})$ & $\begin{array}{l}\text { I-hydroxyphenanthrene } \\
(\mu \mathrm{g} / \mathrm{L})\end{array}$ & $\begin{array}{l}\text { 2-hydroxyfluorene } \\
(\mu \mathrm{g} / \mathrm{L})\end{array}$ \\
\hline Total & $0.19(0.17-0.21)$ & $3.66(3.26-4.12)$ & $0.11(0.10-0.12)$ & $0.43(0.39-0.47)$ \\
\hline \multicolumn{5}{|l|}{ Age group } \\
\hline $19-29 \mathrm{yr}^{\mathrm{a}}$ & $0.15(0.13-0.17)$ & $2.99(2.45-3.65)$ & $0.09(0.08-0.10)$ & $0.37(0.32-0.43)$ \\
\hline $30-39 \mathrm{yr}$ & $0.15(0.14-0.17)$ & $3.21(2.73-3.77)$ & $0.10(0.09-0.11)^{*}$ & $0.37(0.33-0.43)$ \\
\hline $40-49 \mathrm{yr}$ & $0.20(0.18-0.23)^{* * *}$ & $3.78(3.26-4.39)^{* *}$ & $0.11(0.10-0.13)^{* * *}$ & $0.46(0.40-0.53)^{* *}$ \\
\hline $50-59 \mathrm{yr}$ & $0.21(0.19-0.24)^{* * * *}$ & $3.84(3.33-4.42)^{* *}$ & $0.12(0.11-0.14)^{* * *}$ & $0.50(0.44-0.56)^{* * *}$ \\
\hline $60-69 \mathrm{yr}$ & $0.22(0.20-0.25)^{* * *}$ & $3.81(3.24-4.48)^{*}$ & $0.12(0.10-0.13)^{* * *}$ & $0.45(0.39-0.5 \mathrm{I})^{* *}$ \\
\hline$\geq 70 \mathrm{yr}$ & $0.21(0.19-0.24)^{* * *}$ & $4.55(3.76-5.51)^{* *}$ & $0.11(0.10-0.13)^{* * *}$ & $0.42(0.37-0.48)^{*}$ \\
\hline \multicolumn{5}{|l|}{ BMI group } \\
\hline Underweight & $0.21(0.16-0.27)$ & $4.20(3.17-5.55)$ & $0.10(0.08-0.12)$ & $0.45(0.35-0.57)$ \\
\hline Normal $^{\mathrm{a}}$ & $0.18(0.16-0.19)$ & $3.49(3.07-3.97)$ & $0.11(0.10-0.12)$ & $0.39(0.35-0.43)$ \\
\hline Overweight & $0.19(0.17-0.21)$ & $3.48(3.05-3.97)$ & $0.11(0.10-0.12)$ & $0.43(0.39-0.47)$ \\
\hline Obese & $0.19(0.17-0.20)$ & $3.52(3.16-3.93)$ & $0.11(0.11-0.12)$ & $0.44(0.40-0.48)^{*}$ \\
\hline \multicolumn{5}{|c|}{ Household income (USD/month) } \\
\hline$<920$ USD & $0.19(0.16-0.21)$ & $3.85(3.25-4.55)$ & $0.10(0.09-0.12)$ & $0.43(0.38-0.48)$ \\
\hline 920-2090 USD & $0.20(0.17-0.22)$ & $3.67(3.17-4.24)$ & $0.11(0.09-0.12)$ & $0.42(0.37-0.48)$ \\
\hline 2090-3400 USD & $0.19(0.17-0.21)$ & $3.70(3.22-4.25)$ & $0.11(0.10-0.12)$ & $0.43(0.38-0.48)$ \\
\hline$\geq 3400$ USD $^{a}$ & $0.19(0.17-0.22)$ & $3.44(3.01-3.93)$ & $0.11(0.10-0.12)$ & $0.43(0.38-0.48)$ \\
\hline \multicolumn{5}{|c|}{ Alcohol consumption } \\
\hline Never $^{\mathrm{a}}$ & $0.18(0.16-0.21)$ & $3.67(3.17-4.25)$ & $0.11(0.10-0.12)$ & $0.43(0.38-0.48)$ \\
\hline Former & $0.19(0.16-0.22)$ & $3.68(3.03-4.47)$ & $0.11(0.09-0.12)$ & $0.43(0.36-0.50)$ \\
\hline Current & $0.20(0.18-0.22)$ & $3.64(3.23-4.09)$ & $0.11(0.10-0.12)$ & $0.43(0.39-0.47)$ \\
\hline \multicolumn{5}{|c|}{ Regular physical activity } \\
\hline $\mathrm{No}^{\mathrm{a}}$ & $0.19(0.17-0.21)$ & $2.38(2.20-2.59)$ & $0.10(0.09-0.11)$ & $0.42(0.38-0.46)$ \\
\hline Yes & $0.19(0.17-0.22)$ & $2.51(2.30-2.74)$ & $0.11(0.10-0.12)^{*}$ & $0.43(0.39-0.48)$ \\
\hline \multicolumn{5}{|l|}{ Diabetes mellitus } \\
\hline $\mathrm{No}^{\mathrm{a}}$ & $0.18(0.16-0.20)$ & $3.26(2.96-3.60)$ & $0.10(0.10-0.11)$ & $0.40(0.37-0.43)$ \\
\hline Yes & $0.20(0.18-0.23)$ & $4.11(3.40-4.97)^{*}$ & $0.11(0.10-0.13)$ & $0.45(0.39-0.52)^{*}$ \\
\hline
\end{tabular}

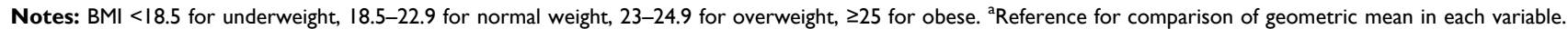
Adjusted for log-transformed urine creatinine, log-transformed urine cotinine, and all variables in the table. $* * * P<0.001 ; * * P<0.01 ; * P<0.05$.

Abbreviations: $\mathrm{BMI}$, body mass index; $\mathrm{Cl}$, confidence interval; USD, US dollars.

major environmental sources of PAHs are tobacco smoking, urban air pollution, and occupational exposure. Many previous studies have shown significantly elevated levels of urinary PAHs among coal-handling workers or motor vehicle drivers, and the urinary 1-OHP levels are widely used as a biomarker of PAH exposure. ${ }^{24-26}$ Therefore, the higher levels of PAHs in men can be attributed to their higher smoking rates and occupational exposure compared to women. ${ }^{27-29}$

Older men ( $\geq 40$ years) had higher levels of all four PAHs than their younger counterparts ( $\leq 39$ years). Exposure to PAHs is primarily through breathing ambient air, and PAHs can be eliminated by bronchial clearance. ${ }^{30}$ Impaired removal of contaminated particles by the mucociliary system may enhance the penetration of particles into the bronchial epithelial cells where PAHs are metabolized. ${ }^{30}$ Most PAHs are excreted from the body shortly after exposure. However, small amounts are known to be retained in body fat and the liver, which may induce bioaccumulation of PAHs in the body over time. ${ }^{31}$ These mechanisms may explain the higher PAH exposure in the older Korean population. As this agerelated relationship has not been consistently observed in other ethnic populations, ${ }^{32,33}$ further studies are needed to reveal this finding. 
Table 4 Adjusted Geometric Mean of Polycyclic Aromatic Hydrocarbons ( $\mu \mathrm{g} / \mathrm{L})$ Stratified by Participants Characteristics in Women

\begin{tabular}{|c|c|c|c|c|}
\hline \multirow[t]{2}{*}{ Factors } & \multicolumn{4}{|c|}{ Adjusted Geometric Mean (95\% Cl) } \\
\hline & I-hydroxypyrene $(\mu \mathrm{g} / \mathrm{L})$ & 2-naphthol $(\mu \mathrm{g} / \mathrm{L})$ & $\begin{array}{l}\text { I-hydroxyphenanthrene } \\
\text { ( } \mu \mathrm{g} / \mathrm{L})\end{array}$ & $\begin{array}{l}\text { 2-hydroxyfluorene } \\
(\mu \mathrm{g} / \mathrm{L})\end{array}$ \\
\hline Total & $0.13(0.12-0.14)$ & $1.64(1.45-1.86)$ & $0.09(0.08-0.10)$ & $0.19(0.17-0.21)$ \\
\hline \multicolumn{5}{|l|}{ Age group } \\
\hline $19-29 \mathrm{yr}^{\mathrm{a}}$ & $0.12(0.10-0.14)$ & $1.88(1.48-2.39)$ & $0.08(0.07-0.09)$ & $0.19(0.16-0.23)$ \\
\hline $30-39 \mathrm{yr}$ & $0.11-(0.10-0.13)$ & $1.51(1.25-1.83)^{*}$ & $0.08(0.07-0.10)$ & $0.19(0.17-0.23)$ \\
\hline $40-49 \mathrm{yr}$ & $0.13(0.11-0.14)$ & $1.57(1.32-1.88)$ & $0.09(0.08-0.10)$ & $0.20(0.17-0.23)$ \\
\hline $50-59 \mathrm{yr}$ & $0.13(0.12-0.15)$ & $1.70(1.44-2.02)$ & $0.09(0.08-0.10)$ & $0.19(0.17-0.22)$ \\
\hline $60-69 \mathrm{yr}$ & $0.15(0.13-0.18)$ & $1.54(1.26-1.90)$ & $0.09(0.08-0.1 \mathrm{I})$ & $0.19(0.17-0.22)$ \\
\hline$\geq 70 \mathrm{yr}$ & $0.14(0.12-0.16)$ & $1.66(1.33-2.07)$ & $0.09(0.08-0.10)$ & $0.18(0.15-0.21)$ \\
\hline \multicolumn{5}{|l|}{ BMI group } \\
\hline Underweight & $0.15(0.12-0.18)^{*}$ & $1.55(1.25-1.92)$ & $0.10(0.08-0.12)^{*}$ & $0.22(0.18-0.26)^{*}$ \\
\hline Normal $^{\mathrm{a}}$ & $0.12(0.11-0.13)$ & $1.64(|.4|-1.9 \mid)$ & $0.08(0.07-0.09)$ & $0.18(0.16-0.20)$ \\
\hline Overweight & $0.12(0.11-0.13)$ & $1.63(1.37-1.93)$ & $0.08(0.07-0.09)$ & $0.18(0.16-0.20)$ \\
\hline Obese & $0.13(0.12-0.15)^{*}$ & $1.76(1.54-2.00)$ & $0.09(0.08-0.10)^{* *}$ & $0.19(0.17-0.21)$ \\
\hline \multicolumn{5}{|l|}{ Household income (USD/month) } \\
\hline$<920$ USD & $0.13(0.11-0.14)$ & $1.73(1.44-2.07)$ & $0.09(0.08-0.10)$ & $0.19(0.16-0.21)$ \\
\hline $920-2090$ USD & $0.14(0.12-0.15)$ & $1.77(1.51-2.09)$ & $0.09(0.08-0.10)$ & $0.20(0.17-0.22)$ \\
\hline 2090-3400 USD & $0.13(0.11-0.14)$ & $1.54(1.30-1.82)$ & $0.09(0.08-0.10)$ & $0.20(0.18-0.22)$ \\
\hline$\geq 3400$ USD $^{a}$ & $0.13(0.12-0.15)$ & $1.54(1.34-1.77)$ & $0.09(0.08-0.10)$ & $0.18(0.16-0.21)$ \\
\hline \multicolumn{5}{|l|}{ Alcohol consumption } \\
\hline Never $^{\mathrm{a}}$ & $0.13(0.11-0.14)$ & $1.84(1.64-2.07)$ & $0.09(0.08-0.10)$ & $0.19(0.17-0.21)$ \\
\hline Former & $0.13(0.11-0.15)$ & $1.44(1.1 \mathrm{I}-1.88)$ & $0.09(0.07-0.1 \mathrm{I})$ & $0.20(0.16-0.24)$ \\
\hline Current & $0.14(0.12-0.15)$ & $1.66(1.48-1.87)$ & $0.09(0.08-0.09)$ & $0.18(0.17-0.20)$ \\
\hline \multicolumn{5}{|l|}{ Regular physical activity } \\
\hline $\mathrm{No}^{\mathrm{a}}$ & $0.13(0.12-0.14)$ & $1.59(1.40-1.80)$ & $0.09(0.08-0.10)$ & $0.19(0.17-0.22)$ \\
\hline Yes & $0.13(0.12-0.15)$ & $1.70(1.46-1.97)$ & $0.09(0.08-0.10)$ & $0.19(0.17-0.21)$ \\
\hline \multicolumn{5}{|l|}{ Menopause } \\
\hline No & $0.13(0.12-0.15)$ & $1.61(1.38-1.89)$ & $0.08(0.07-0.09)$ & $0.18(0.16-0.20)$ \\
\hline Yes $^{\mathrm{a}}$ & $0.13(0.11-0.14)$ & $1.67(1.43-1.95)$ & $0.09(0.08-0.10)$ & $0.20(0.18-0.23)$ \\
\hline \multicolumn{5}{|l|}{ Diabetes mellitus } \\
\hline $\mathrm{No}^{\mathrm{a}}$ & $0.14(0.12-0.15)$ & $0.56(1.43-1.70)$ & $0.09(0.08-0.10)$ & $0.19(1.04-0.17)$ \\
\hline Yes & $0.13(0.11-0.14)$ & I.8I (I.54-2.12)* & $0.09(0.08-0.10)$ & $0.20(1.07-0.18)$ \\
\hline
\end{tabular}

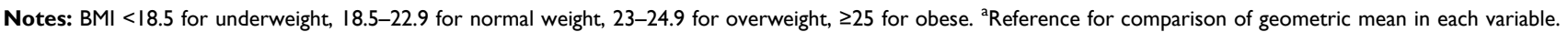
Adjusted for log-transformed urine creatinine, log-transformed urine cotinine, and all variables in the table. $* * P<0.01 ; * P<0.05$.

Abbreviations: $\mathrm{BMI}$, body mass index; $\mathrm{Cl}$, confidence interval; USD, US dollars.

Participants who performed regular physical activity had higher levels of 1-OHPhe than those who did not. 1-OHPhe is a human metabolite of phenanthrene that can be found in the urine of individuals exposed to phenanthrene. ${ }^{34}$ Phenanthrene is detected in particulate emissions from burning natural gas and municipal incinerator waste and in particulates present in atmospheric air pollution near heavy vehicle traffic and manufacturing areas. ${ }^{35}$ During aerobic activity, respiratory absorption of airborne pollutants increases with increase in minute ventilation and diffusion capacity in the lungs. ${ }^{36}$ Although a direct evidence showing higher PAH exposure during the exercise has not been found in human studies, previous epidemiologic studies have demonstrated that habitual exercise in highly pollutant outdoor areas or indoor environments, such as schools, may increase the amount of exposure to air pollutants. ${ }^{37,38}$ Thus, increased urinary 1-OHPhe levels in individuals who performed regular 
Table 5 Adjusted Odds Ratio $(95 \% \mathrm{Cl})$ for Diabetes Mellitus According to the Quartile of Urinary Polycyclic Aromatic Hydrocarbons $(\mu \mathrm{g} / \mathrm{L})$

\begin{tabular}{|c|c|c|c|c|c|}
\hline & \multicolumn{4}{|c|}{ Adjusted Odds Ratio $(95 \% \mathrm{Cl})$} & \multirow[t]{2}{*}{ P-trend } \\
\hline & I & II & III & IV & \\
\hline \multicolumn{6}{|c|}{ I-hydroxypyrene } \\
\hline Total & 1.00 & $0.98(0.66-1.46)$ & $0.85(0.55-1.31)$ & $1.02(0.62-1.7 \mid)$ & 0.963 \\
\hline Men & 1.00 & $1.35(0.73-2.50)$ & $0.91(0.49-1.68)$ & $1.50(0.70-3.20)$ & 0.455 \\
\hline Women & 1.00 & $0.79(0.49-1.29)$ & $0.84(0.48-1.48)$ & $0.74(0.38-1.43)$ & 0.427 \\
\hline \multicolumn{6}{|l|}{ 2-naphthol } \\
\hline Total & 1.00 & $1.08(0.79-1.49)$ & $1.57(1.09-2.27)$ & $1.83(1.29-2.60)$ & $<0.001$ \\
\hline Men & 1.00 & $0.91(0.56-1.50)$ & $1.27(0.73-2.27)$ & $2.07(1.20-3.55)$ & 0.013 \\
\hline Women & 1.00 & $1.43(0.89-2.29)$ & $2.3 \mathrm{I}(\mathrm{I} .4 \mathrm{I}-3.8 \mathrm{I})$ & $1.94(1.18-3.18)$ & 0.002 \\
\hline \multicolumn{6}{|c|}{ I-hydroxyphenanthrene } \\
\hline Total & 1.00 & $0.89(0.59-1.36)$ & $1.02(0.64-1.63)$ & $0.77(0.47-1.25)$ & 0.936 \\
\hline Men & 1.00 & $0.8 \mathrm{I}(0.4 \mathrm{I}-1.59)$ & $0.73(0.34-1.57)$ & $0.78(0.36-1.67)$ & 0.918 \\
\hline Women & 1.00 & $1.04(0.62-1.75)$ & $1.40(0.82-2.40)$ & $0.7 \mid(0.37-1.36)$ & 0.425 \\
\hline \multicolumn{6}{|c|}{ 2-hydroxyfluorene } \\
\hline Total & 1.00 & $1.40(0.95-2.05)$ & $1.21(0.78-1.90)$ & $1.81(1.10-2.98)$ & 0.064 \\
\hline Men & 1.00 & $1.59(0.88-2.88)$ & $1.29(0.64-2.62)$ & $2.19(0.92-5.24)$ & 0.212 \\
\hline Women & 1.00 & $1.28(0.79-2.02)$ & $1.26(0.69-2.29)$ & $1.90(0.98-3.7 \mathrm{I})$ & 0.078 \\
\hline
\end{tabular}

Notes: Odds ratios were adjusted for sex, age group, body mass index group, household income, alcohol consumption, physical activity, log-transformed urinary creatinine and cotinine, menopausal status (in women), and quartiles of all other PAHs.

Abbreviation: $\mathrm{Cl}$, confidence interval.

physical activity may reflect increased inhalation of outdoor or indoor phenanthrene during the exercise through the aforementioned manner. Further epidemiologic and experimental studies are required to disclose this finding.

In this study, Korean adults with diabetes showed higher urinary 2-NAP levels in both sexes than those without. We also found that higher urinary 2-NAP quartiles were significantly associated with an increased OR for diabetes in a dose-dependent manner. The highest quartile had a higher OR for diabetes $(1.83,95 \%$ CI 1.29-2.60) than the lowest quartile. Similarly, previous studies using the NHANES data have found elevated ORs $(1.42-3.30)$ in adults with higher 2-NAP exposure. ${ }^{11,12,14}$ Meanwhile, a study from China showed a higher likelihood of diabetes in individuals with higher 2-NAP exposure, but the difference was not statistically significant. ${ }^{39}$

We found significantly higher urinary 2-OHFlu levels in men with diabetes than in those without. Particularly, the urinary 2-OHFlu levels were positively associated with diabetes (OR 1.81, 95\% CI 1.10-2.98), although the significance was lost in the sex-specific analysis. This positive association between 2-OHFlu exposure and diabetes has been reported in previous studies; ${ }^{11,39}$ while other studies have found no statistically significant associations. ${ }^{12,14}$
We could not find any significant association between the 1-OHP and 1-OHPhe levels and diabetes, which is inconsistent with previous studies showing positive associations of 1-OHP ${ }^{12,14}$ and 1-OHPhe ${ }^{39}$ exposure with diabetes. This discrepancy might be partly due to the lower urinary levels of 1-OHP and 1-OHPhe in Korea than in other countries. ${ }^{11-14,16-18,39}$

Besides the four PAH metabolites mentioned in this study, other studies have shown a link between diabetes and other PAHs, which were not analyzed in Korea, such as 1-NAP, ${ }^{12,14}$ 9-OHFlu, ${ }^{11,39}$ 2-OHPhe, ${ }^{11,12,14,39}$ 4-OHPhe, ${ }^{39}$ and 9-OHPhe. ${ }^{39}$ The KoNEHS currently examines only the four types of metabolites; therefore, analyzing the different types of PAHs and verifying their mixture effects would be necessary in further studies.

Although a causal relationship is not clear so far, several plausible mechanisms regarding the association between PAHs and diabetes are suggested. First, PAHs are known to enhance the generation of reactive oxygen species ${ }^{40}$ by inducing aryl hydrocarbon receptor-dependent gene expression. ${ }^{41}$ Oxidative stress plays a critical role in systemic inflammation and impairs the function of $\beta$-cells, resulting in insulin resistance. ${ }^{7,42,43}$ Second, PAHs can disrupt peroxisome proliferator-activated receptors (PPARs), ${ }^{44}$ 
which are major regulators of adipogenesis and glucose metabolism. Third, PAHs influence epigenetic modification, such as defects in DNA methylation. PAHs interfere with DNA methylation during pancreatic $\beta$-cell development ${ }^{9}$ and DNA methylation of insulin receptor substrate $2^{10}$ and PPAR- $\gamma .{ }^{45}$ Other mechanisms include hepatic dysfunction, ${ }^{41}$ skeletal muscle inflammation, and endothelial dysfunction, which reduce peripheral glucose uptake. $^{46,47}$ To summarize, PAHs may directly impair $\beta$-cell function through oxidative stress and systemic inflammation, and indirectly induce diabetes by adipose tissue dysregulation.

An important feature of this study is that we analyzed creatinine level by adjustment rather than division with the PAH level. When urinary dilution is adjusted by merely dividing the PAH levels by the creatine levels, the study results might be greatly affected by factors involved with creatinine levels, such as kidney function, BMI, and age. $^{48,49}$ We also used the urinary cotinine levels as covariates to reduce the smoking effect on diabetes, as smoking is a major contributor of PAH exposure. Besides, the risk of diabetes could be affected by the endogenous estrogen status; hence, we included the menopausal status in women as a covariate. Furthermore, as PAHs showed positive correlations with diabetes, we adjusted for all other PAH levels to see the individual effects of each PAH.

Our study had some limitations. We did not assess the possible routes of exposure other than smoking. Although second-hand smoke exposure was not investigated, urine cotinine concentrations were adjusted in all statistics to overcome this limitation. Due to the relatively short half-lives of PAHs, the spot urine PAH levels may reflect only recent and not past or long-term exposure. Additionally, the diagnosis of diabetes was based only on a questionnaire survey and not on biochemical studies, such as a fasting blood glucose or glycosylated hemoglobin test. Moreover, the KoNEHS data did not differentiate the types of diabetes (ie, whether the participants have type 1 or type 2 diabetes). Finally, because of the crosssectional nature of the study, we could not draw a causal relationship between PAHs and diabetes mellitus. Despite these limitations, this is the first study to investigate the association between $\mathrm{PAH}$ exposure and diabetes in a large population, with adjustment for various confounding factors, such as sex, age, BMI, household income, alcohol consumption, physical activity, urinary creatinine and cotinine, menopausal status (in women), and quartiles of all other PAHs.

\section{Conclusion}

This study revealed that the urinary 2-NAP and 2-OHFlu levels were associated with diabetes mellitus in Korean adults. Given the ongoing rise in diabetes prevalence, our findings may have considerable implications for public health. To understand the mechanism between PAH exposure and diabetes mellitus, further longitudinal studies including analyses of exposure sources, air pollution data, and the mixture effects of environmental pollutants, are warranted.

\section{Funding}

There is no funding to report.

\section{Disclosure}

The authors report no conflict of interest in this work.

\section{References}

1. Chevalier N, Fénichel P. Endocrine disruptors: new players in the pathophysiology of type 2 diabetes? Diabetes Metab. 2015;41 (2):107-115. doi:10.1016/j.diabet.2014.09.005

2. Cho NH, Shaw JE, Karuranga S, et al. IDF diabetes atlas: global estimates of diabetes prevalence for 2017 and projections for 2045. Diabetes Res Clin Pract. 2018;138:271-281. doi:10.1016/j.diabres.2018.02.023

3. Won JC, Lee JH, Kim JH, et al. Diabetes fact sheet in Korea, 2016: an appraisal of current status. Diabetes Metab J. 2018;42 (5):415-424. doi:10.4093/dmj.2018.0017

4. Wu Y, Ding Y, Tanaka Y, Zhang W. Risk factors contributing to type 2 diabetes and recent advances in the treatment and prevention. Int J Med Sci. 2014;11(11):1185-1200. doi:10.7150/ijms.10001

5. Abdel-Shafy H, Mansour M. A review on polycyclic aromatic hydrocarbons: source, environmental impact, effect on human health and remediation. Egypt $J$ Petrol. 2016;25:107-123. doi:10.1016/j. ejpe.2015.03.011

6. Jeng HA, Pan $\mathrm{CH}$, Diawara $\mathrm{N}$, et al. Polycyclic aromatic hydrocarbon-induced oxidative stress and lipid peroxidation in relation to immunological alteration. Occup Environ Med. 2011;68 (9):653-658. doi:10.1136/oem.2010.055020

7. Alshaarawy O, Zhu M, Ducatman A, Conway B, Andrew ME. Polycyclic aromatic hydrocarbon biomarkers and serum markers of inflammation. A positive association that is more evident in men. Environ Res. 2013;126:98-104. doi:10.1016/j.envres.2013.07.006

8. Khalil A, Villard PH, Dao MA, et al. Polycyclic aromatic hydrocarbons potentiate high-fat diet effects on intestinal inflammation. Toxicol Lett. 2010;196(3):161-167. doi:10.1016/j.toxlet.2010.04.010

9. Yun Y, Zhang Y, Li G, Chen S, Sang N. Embryonic exposure to oxypolycyclic aromatic hydrocarbon interfere with pancreatic $\beta$-cell development in zebrafish via altering DNA methylation and gene expression. Sci Total Environ. 2019;660:1602-1609. doi:10.1016/j. scitotenv.2018.12.476

10. Kim YH, Lee YS, Lee DH, Kim DS. Polycyclic aromatic hydrocarbons are associated with insulin receptor substrate 2 methylation in adipose tissues of Korean women. Environ Res. 2016;150:47-51.

11. Stallings-Smith S, Mease A, Johnson TM, Arikawa AY. Exploring the association between polycyclic aromatic hydrocarbons and diabetes among adults in the United States. Environ Res. 2018;166:588-594. doi:10.1016/j.envres.2018.06.041

12. Ranjbar M, Rotondi MA, Ardern CI, Kuk JL. Urinary biomarkers of polycyclic aromatic hydrocarbons are associated with cardiometabolic health risk. PLoS One. 2015;10(9):e0137536. 
13. Hu H, Kan H, Kearney GD, Xu X. Associations between exposure to polycyclic aromatic hydrocarbons and glucose homeostasis as well as metabolic syndrome in nondiabetic adults. Sci Total Environ. 2015;505:56-64. doi:10.1016/j.scitotenv.2014.09.085

14. Alshaarawy O, Zhu M, Ducatman AM, Conway B, Andrew ME. Urinary polycyclic aromatic hydrocarbon biomarkers and diabetes mellitus. Occup Environ Med. 2014;71(6):437-441. doi:10.1136/oemed-2013-101987

15. Wang L, Hou J, Hu C, et al. Mediating factors explaining the associations between polycyclic aromatic hydrocarbons exposure, low socioeconomic status and diabetes: a structural equation modeling approach. Sci Total Environ. 2019;648:1476-1483. doi:10.1016/j. scitotenv.2018.08.255

16. Hou J, Sun H, Zhou Y, et al. Environmental exposure to polycyclic aromatic hydrocarbons, kitchen ventilation, fractional exhaled nitric oxide, and risk of diabetes among Chinese females. Indoor Air. 2018;28(3):383-393. doi:10.1111/ina.12453

17. Yang L, Yan K, Zeng D, et al. Association of polycyclic aromatic hydrocarbons metabolites and risk of diabetes in coke oven workers. Environ Pollut. 2017;223:305-310. doi:10.1016/j.envpol.2017.01.027

18. Hou J, Sun H, Xiao L, et al. Combined effect of urinary monohydroxylated polycyclic aromatic hydrocarbons and impaired lung function on diabetes. Environ Res. 2016;148:467-474. doi:10.1016/ j.envres.2016.03.038

19. Bae S, Kwon HJ. Current state of research on the risk of morbidity and mortality associated with air pollution in Korea. Yonsei Med J. 2019;60(3):243-256. doi:10.3349/ymj.2019.60.3.243

20. Choi W, Kim S, Baek YW, et al. Exposure to environmental chemicals among Korean adults-updates from the second Korean National Environmental Health Survey (2012-2014). Int J Hyg Environ Health. 2017;220(2):29-35. doi:10.1016/j.ijheh.2016.10.002

21. Taskforce IOJMHCAPLIAfSoO. The Asia-Pacific perspective: redefining obesity and its treatment. 2000:8-14.

22. Control CfD, Prevention \%J Washington DC. Fourth National Report on Human Exposure to Environmental Chemicals, Update Tables. 2015.

23. Canada H Fourth Report on Human Biomonitoring of Environmental Chemicals in Canada. 2017.

24. Malkin R, Kiefer M, Tolos W. 1-Hydroxypyrene levels in coal-handling workers at a coke oven. J Occup Environ Med. 1996;38(11):1141-1144. doi:10.1097/00043764-199611000-00014

25. Nguyen TT, Kawanami S, Kawai K, et al. Urinary 1-hydroxypyrene and 8-hydroxydeoxyguanosine levels among coke-oven workers for 2 consecutive days. J Occup Health. 2014;56(3):178-185. doi:10.1539/joh.130222-OA

26. Brucker N, Charão MF, Moro AM, et al. Atherosclerotic process in taxi drivers occupationally exposed to air pollution and co-morbidities. Environ Res. 2014;131:31-38. doi:10.1016/j.envres.2014.02.012

27. Ding YS, Trommel JS, Yan XJ, Ashley D, Watson CH. Determination of 14 polycyclic aromatic hydrocarbons in mainstream smoke from domestic cigarettes. Environ Sci Technol. 2005;39 (2):471-478. doi:10.1021/es048690k

28. Koh DH, Park JH, Lee SG, et al. Comparison of polycyclic aromatic hydrocarbons exposure across occupations using urinary metabolite 1-hydroxypyrene. Ann Work Expo Health. 2020;64(4):445-454. doi:10.1093/annweh/wxaa014

29. Gehle K Toxicity of polycyclic aromatic hydrocarbons (PAHs). 2009.

30. WHO. Guidelines approved by the guidelines review committee. In: WHO Guidelines for Indoor Air Quality: Selected Pollutants. Geneva: World Health Organization; 2010.

31. Obana H, Hori S, Kashimoto T, Kunita N. Polycyclic aromatic hydrocarbons in human fat and liver. Bull Environ Contam Toxicol. 1981;27(1):23-27. doi:10.1007/BF01610981

32. Agudelo-Castañeda DM, Teixeira EC, Schneider IL, Lara SR, Silva LFO. Exposure to polycyclic aromatic hydrocarbons in atmospheric PM(1.0) of urban environments: carcinogenic and mutagenic respiratory health risk by age groups. Environ Pollut. 2017;224:158-170. doi:10.1016/j.envpol.2017.01.075
33. Huang W, Grainger J, Patterson DG, et al. Comparison of 1-hydroxypyrene exposure in the US population with that in occupational exposure studies. Int Arch Occup Environ Health. 2004;77 (7):491-498. doi:10.1007/s00420-004-0529-y

34. Best EA, Juarez-Colunga E, James K, LeBlanc WG, Serdar B. Biomarkers of exposure to polycyclic aromatic hydrocarbons and cognitive function among elderly in the United States (National Health and Nutrition Examination Survey: 2001-2002). PLoS One. 2016;11(2):e0147632.

35. Fang GC, Wu YS, Chen JC, Chang CN, Ho TT. Characteristic of polycyclic aromatic hydrocarbon concentrations and source identification for fine and coarse particulates at Taichung Harbor near Taiwan Strait during 2004-2005. Sci Total Environ. 2006;366(2-3):729-738. doi:10.1016/j.scitotenv.2005.09.075

36. Künzli N. The public health relevance of air pollution abatement. Eur Respir J. 2002;20(1):198-209. doi:10.1183/09031936.02.00401502

37. Slezakova K, Peixoto C, Carmo Pereira MD, Morais S. (Ultra) Fine particle concentrations and exposure in different indoor and outdoor microenvironments during physical exercising. $J$ Toxicol Environ Health A. 2019;82(9):591-602. doi:10.1080/15287394.2019.1636494

38. Braniš M, Safránek J, Hytychová A. Indoor and outdoor sources of size-resolved mass concentration of particulate matter in a school gym-implications for exposure of exercising children. Environ Sci Pollut Res Int. 2011;18(4):598-609. doi:10.1007/ s11356-010-0405-0

39. Yang L, Zhou Y, Sun H, et al. Dose-response relationship between polycyclic aromatic hydrocarbon metabolites and risk of diabetes in the general Chinese population. Environ Pollut. 2014;195:24-30. doi:10.1016/j.envpol.2014.08.012

40. Patri M, Padmini A, Babu P. Polycyclic aromatic hydrocarbons in air and their neurotoxic potency in association with oxidative stress: a brief perspective. Ann Neurosci. 2010;16(1):22-30.

41. Zhang XJ, Shi Z, Lyv JX, He X, Englert NA, Zhang SY. Pyrene is a novel Constitutive Androstane Receptor (CAR) activator and causes hepatotoxicity by CAR. Toxicol Sci. 2015;147(2):436-445. doi:10.1093/toxsci/kfv142

42. Asmat U, Abad K, Ismail K. Diabetes mellitus and oxidative stressA concise review. Saudi Pharm J. 2016;24(5):547-553. doi:10.1016/ j.jsps.2015.03.013

43. Farzan SF, Chen Y, Trachtman H, Trasande L. Urinary polycyclic aromatic hydrocarbons and measures of oxidative stress, inflammation and renal function in adolescents: NHANES 2003-2008. Environ Res. 2016;144(Pt A):149-157. doi:10.1016/j.envres.2015.11.012

44. Kim J-H, Yamaguchi K, Lee S-H, et al. Evaluation of polycyclic aromatic hydrocarbons in the activation of early growth response-1 and peroxisome proliferator activated receptors. Toxicol Sci. 2005;85 (1):585-593. doi:10.1093/toxsci/kfi118

45. Yan Z, Zhang H, Maher C, et al. Prenatal polycyclic aromatic hydrocarbon, adiposity, peroxisome proliferator-activated receptor (PPAR) $\gamma$ methylation in offspring, grand-offspring mice. PLoS One. 2014;9 (10):e110706. doi:10.1371/journal.pone.0110706

46. Rao X, Patel P, Puett R, Rajagopalan S. Air pollution as a risk factor for type 2 diabetes. Toxicol Sci. 2015;143(2):231-241. doi:10.1093/ toxsci/kfu250

47. Esposito K, Petrizzo M, Maiorino MI, Bellastella G, Giugliano D. Particulate matter pollutants and risk of type 2 diabetes: a time for concern? Endocrine. 2016;51(1):32-37. doi:10.1007/s12020-0150638-2

48. Bulka CM, Mabila SL, Lash JP, Turyk ME, Argos M. Arsenic and obesity: a comparison of urine dilution adjustment methods. Environ Health Perspect. 2017;125(8):087020. doi:10.1289/EHP1202

49. O'Brien KM, Upson K, Cook NR, Weinberg CR. Environmental chemicals in urine and blood: improving methods for creatinine and lipid adjustment. Environ Health Perspect. 2016;124(2):220-227. doi:10.1289/ehp.1509693 


\section{Publish your work in this journal}

Diabetes, Metabolic Syndrome and Obesity: Targets and Therapy is an international, peer-reviewed open-access journal committed to the rapid publication of the latest laboratory and clinical findings in the fields of diabetes, metabolic syndrome and obesity research. Original research, review, case reports, hypothesis formation, expert opinion and commentaries are all considered for publication. The manuscript management system is completely online and includes a very quick and fair peer-review system, which is all easy to use. Visit http://www.dovepress.com/testimonials.php to read real quotes from published authors.

Submit your manuscript here: https://www.dovepress.com/diabetes-metabolic-syndrome-and-obesity-targets-and-therapy-journal 